\title{
Biomarkers and key pathways in atrial fibrillation associated with mitral valve disease identified by multi-omics study
}

\author{
Ming-Yang Li ${ }^{1}$, Huan-Xin Chen ${ }^{1}$, Hai-Tao Hou ${ }^{1}$, Jun Wang ${ }^{1}$, Xiao-Cheng Liu ${ }^{1}$, Qin Yang ${ }^{1}$, \\ Guo-Wei He $\mathrm{He}^{1,2,3,4}$ ^
}

${ }^{1}$ Center for Basic Medical Research \& Department of Cardiovascular Surgery, TEDA International Cardiovascular Hospital, Chinese Academy of Medical Sciences \& Graduate School, Peking Union Medical College, Beijing, China; ${ }^{2}$ The Institute of Cardiovascular Diseases, Tianjin University, Tianjin, China; ${ }^{3}$ Drug Research and Development Center, Wannan Medical College, Wuhu, China; ${ }^{4}$ Department of Surgery, Oregon Health and Science University, Portland, OR, USA

Contributions: (I) Conception and design: GW He; (II) Administrative support: GW He, J Wang, XC Liu; (III) Provision of study materials or patients: GW He, MY Li, HX Chen, HT Hou, J Wang, XC Liu; (IV) Collection and assembly of data: MY Li, HX Chen, GW He; (V) Data analysis and interpretation: MY Li, GW He, HX Chen, HT Hou, Q Yang; (VI) Manuscript writing: All authors; (VII) Final approval of manuscript: All authors.

Correspondence to: Prof. Guo-Wei He, MD, PhD, DSc. Center for Basic Medical Research \& Department of Cardiovascular Surgery, TEDA International Cardiovascular Hospital, Chinese Academy of Medical Sciences \& Peking Union Medical College, Tianjin, China. Email: gwhezj@163.com.

Background: Mitral valve disease (MVD)-associated atrial fibrillation (AF) is one of the most common arrhythmias with an increased risk of thromboembolic events. This study aimed to identify the molecular mechanisms and possible biomarkers for chronic AF in MVD by using multi-omics methods.

Methods: This prospective study enrolled patients with MVD ( $=100)$ undergoing mitral valve replacement surgery. The patients were allocated into chronic AF and sinus rhythm (SR) groups. Plasma samples were collected preoperatively. Proteomics was performed with isobaric tags for relative and absolute quantitation (iTRAQ) to identify differential proteins (DPs) between the two groups. The selected DPs were then validated in a new cohort of patients by enzyme-linked immunosorbent assay (ELISA). A gas chromatography-mass spectrometer was used in the metabolomics study to identify differential metabolites (DMs). Bioinformatics analyses were performed to analyze the results.

Results: Among the 447 plasma proteins and 322 metabolites detected, 57 proteins and 55 metabolites, including apolipoprotein A-I (ApoA-I), apolipoprotein A-II (ApoA-II), LIM domain only protein 7 (LMO7), and vitronectin $(\mathrm{VN})$ were differentially expressed between $\mathrm{AF}$ and $\mathrm{SR}$ patients. Bioinformatics analyses identified enriched pathways related to AF, including peroxisome proliferator-activated receptor alpha $(\mathrm{PPAR} \alpha)$, the renin angiotensin aldosterone system (RAAS), galactose, biosynthesis of unsaturated fatty acids, and linoleic acid metabolism.

Conclusions: Using integrated multi-omics technologies in MVD-associated AF patients, the present study, for the first time, revealed important signaling pathways, such as PPAR $\alpha$, as well as possible roles of other signaling pathways, including the RAAS and galactose metabolism to understand the molecular mechanism of MVD-associated AF. It also identified a large number of DPs and DMs. Some identified proteins and metabolites, such as ApoA-I, ApoA-II, LMO7, and VN, may be further developed as biomarkers for MVD-associated AF.

Keywords: Atrial fibrillation (AF); mitral valve disease (MVD); proteomics; metabolomics

\footnotetext{
^ ORCID: 0000-0001-6497-876X.
} 
Submitted May 07, 2020. Accepted for publication Nov 06, 2020.

doi: $10.21037 /$ atm-20-3767

View this article at: http://dx.doi.org/10.21037/atm-20-3767

\section{Introduction}

Atrial fibrillation (AF) is one of the most common arrhythmias $(1,2)$. It is known that electrical, contractile, and structural remodeling play important roles in the initiation and maintenance of AF (3). Patients with mitral valve disease (MVD) and AF are associated with an increased risk of thromboembolic events. A recent study (4) reported that the prevalence of persistent or permanent AF in MVD is as high as $87.1 \%$ in mitral regurgitation and $86.6 \%$ in aortic regurgitation, highlighting the importance of $\mathrm{AF}$ in MVD. Despite efforts to discover plasma biomarkers in MVD (5) and to identify the role of ion channels (6), the molecular mechanism of AF in MVD remains unclear. Recently, proteomics studies were performed to explore the mechanisms of AF (6-10). These studies identified the differential proteins (DPs) in the right and left atrial appendage of rheumatic MVD patients $(6,7,9)$, or pathways including ketone body (8) or glutathione (10) metabolism and the peroxisome proliferator-activated receptor (PPAR) pathway $(9,10)$, which are related to postoperative $\mathrm{AF}(8-10)$.

We recently performed multi-omics studies in MVD $(5,6,9)$, congenital heart disease (11-14), and coronary artery disease (15). In human atrial tissues from patients with rheumatic MVD in persistent AF compared to sinus rhythm (SR) using a combined transcriptomic and proteomic approach, we found that intracellular chloride channels (6) as well as PPAR $\alpha, \beta$, and $\gamma$ pathways play an important role in the development of AF (9). However, for persistent and chronic AF in MVD, no combined proteomics and metabolomics studies have been reported. In this study, we used multi-omics methods to investigate plasma samples from patients with MVD in AF or in SR undergoing heart valve replacement surgery to identify pathways and biomarkers associated with $\mathrm{AF}$. We present the following article following the Strengthening the Reporting of Observational Studies in Epidemiology (STROBE) reporting checklist (available at http://dx.doi.org/10.21037/atm-20-3767).

\section{Methods}

\section{Patient recruitment and sample collection}

From January 2013 to December 2017, 100 consecutive patients with MVD who underwent mitral valve replacement surgery at TEDA International Cardiovascular Hospital were enrolled and grouped into either chronic AF $(n=70)$ or SR $(n=30)$. AF was diagnosed by history, physical examination, and a 12-lead electrocardiogram. For comparison, patients in SR were screened to ensure that they had no AF by direct questioning about symptoms suggestive of AF and by analysis of 12-lead electrocardiograms. Patients with infective endocarditis, sick sinus syndrome, familial paroxysmal AF (lone AF/familial AF), pulmonary heart disease, cardiomyopathy, hyperthyroidism, chronic kidney diseases, coronary artery disease, and those under the age of 18 years in both groups were also excluded to reduce prevalence-incidence bias. The demographic characteristics of patients are shown in Tables S1,S2.

This study was approved by the Ethics Committee of the TEDA International Cardiovascular Hospital \{No. [2019]0315-1\}. Written informed consent was obtained from all patients enrolled in the study and conforms to the principles outlined in the Declaration of Helsinki (as revised in 2013). Blood samples were collected from all patients the day before surgery and were then centrifuged at 3,500 rpm for $10 \mathrm{~min}$ to separate the plasma from blood cells.

\section{Proteomics analysis}

A discovery-validation workflow was utilized in the proteomics analysis, and isobaric tags for relative and absolute quantitation (iTRAQ) were used for the proteomics study. As eight isobaric tags ( $\mathrm{m} / \mathrm{z} 113,114,115$, $116,117,118,119$, and 121) were applied in the study, the plasma samples from 24 patients were divided into six groups (three AF groups and three SR groups). Each of these groups contained the plasma samples from four patients. Also, plasma samples from $12 \mathrm{AF}$ or $12 \mathrm{SR}$ patients were used as controls (Figure S1). Thus, the eight isobaric tags were concomitantly labeled in the plasma samples for the iTRAQ study (16).

In the validation phase, DPs identified by the above iTRAQ study were validated in plasma samples taken from a new cohort of patients using enzyme-linked immunosorbent assays (ELISA). 


\section{Metabolomics analysis}

Plasma samples were thawed at room temperature and were then prepared for gas chromatography-mass spectrometry (GC-MS) analysis (Figure S1). ChromaTOF software (v4.34, LECO, St. Joseph, MI, USA) and the Fiehn database were used to process the raw data and to provide qualitative information about the metabolites. The Kyoto Encyclopedia of Genes and Genomes (KEGG) pathway database was used to generate significant pathways in the metabolomics dataset (17).

\section{Creation of pathway maps}

All of the omics data were used to compare proteins/ enzymes with metabolic pathways. Quantitative changes of individual metabolites and proteins were calculated and graphed using the KEGG mapping tools. By combining proteomics and metabolomics data, we were able to identify the common pathways involved in $\mathrm{AF}$.

\section{Statistical analysis}

SPSS 23.0 software (SPSS Inc., Chicago, IL, USA) and GraphPad 6.0c software (GraphPad Software, La Jolla, CA, USA) were used for statistical analysis. Data are expressed as mean \pm standard error of the mean (SEM). Comparisons of statistical data were performed using either the independent samples $t$-test or Chi-square tests. A $\mathrm{P}$ value of $<0.05$ was considered statistically significant.

More detailed methods are presented in Appendix 1.

\section{Results}

\section{Patient characteristics}

The clinical characteristics of the study cohorts are summarized in Tables S1,S2. Patients in the AF group presented with larger left atrium-systolic diameters (LA-Ds), right ventricular-diastolic diameters (RV-Dd), right atriumsystolic diameters (RA-Ds), and lower left ventricular ejection fraction (LVEF) than those in the SR group in both iTRAQ (Table S1) and ELISA validation (Table S2).

\section{Proteomics}

A large number of unique peptides were detected by iTRAQ. By matching this data with the theoretical spectrum, 447 reliable proteins were identified. Under the condition of fold change (FC) $>1.2$ or $<0.83$ and $\mathrm{P}$ value $\leq 0.05$, the number of differentially expressed proteins in the AF group was 57 (Table S3, Figure 1A), including 33 up-regulated and 24 down-regulated (https://cdn.amegroups.cn/static/public/ atm-20-3767-1.xlsx).

Bioinformatics analyses including Gene Ontology (GO) function and enrichment, KEGG pathway, and proteinprotein interaction (PPI) were also performed (https://cdn. amegroups.cn/static/public/atm-20-3767-1.xlsx), and the results are shown in Figure 1B,C,D,E. Biological processes such as immune response $(72.7 \%)$, defense response $(74.5 \%)$, receptor-mediated endocytosis $(50.9 \%)$, protein activation cascade $(56.3 \%)$, and complement activation (52.7\%) were enriched. Cellular component enrichment analysis revealed that most of the differentially expressed proteins were from the extracellular region (94.7\%) and that binding $(96.3 \%)$ was their main molecular function. Furthermore, KEGG pathway analysis demonstrated several pathways, such as the PPAR signaling pathway, renin-angiotensin system, complement and coagulation cascades, cell adhesion molecules, and adherent junction, were enriched. The PPI is shown in Figure $1 E$.

\section{Protein validation by ELISA}

Ten proteins were selected to validate the proteomics result by ELISA: apolipoprotein A-I (ApoA-I), apolipoprotein A-II (ApoA-II), LIM domain only protein 7 (LMO7), prothrombin fragment $1+2$, vitronectin $(\mathrm{VN})$, angiotensinogen, ficolin-3, serum amyloid $\mathrm{P}$-component (SAP), macrophage colony-stimulating factor 1 receptor (CSF-1R), and purine nucleoside phosphorylase (PNP) (Figure 2). Six were successfully validated. Of these, the concentration of ApoA-I, angiotensinogen, CSF$1 \mathrm{R}$, and VN 2 was lower in the AF group than those in the SR group, whereas the concentration of LMO7 and prothrombin fragment $1+2$ was higher.

\section{Metabolomics analysis in chronic AF}

In the comparison of the plasma metabolite profile between $30 \mathrm{AF}$ patients and $26 \mathrm{SR}$ patients, 322 metabolites were discovered, including 55 differential metabolites (DMs) (Table S4) between AF and SR patients under the condition of variable importance in projection (VIP) $>1$ in the partial least-squares discriminant analysis (PLS-DA) model and $\mathrm{P}$ values $<0.05$ (https://cdn.amegroups.cn/static/public/ atm-20-3767-2.xlsx). The heatmap of these metabolites is 
A

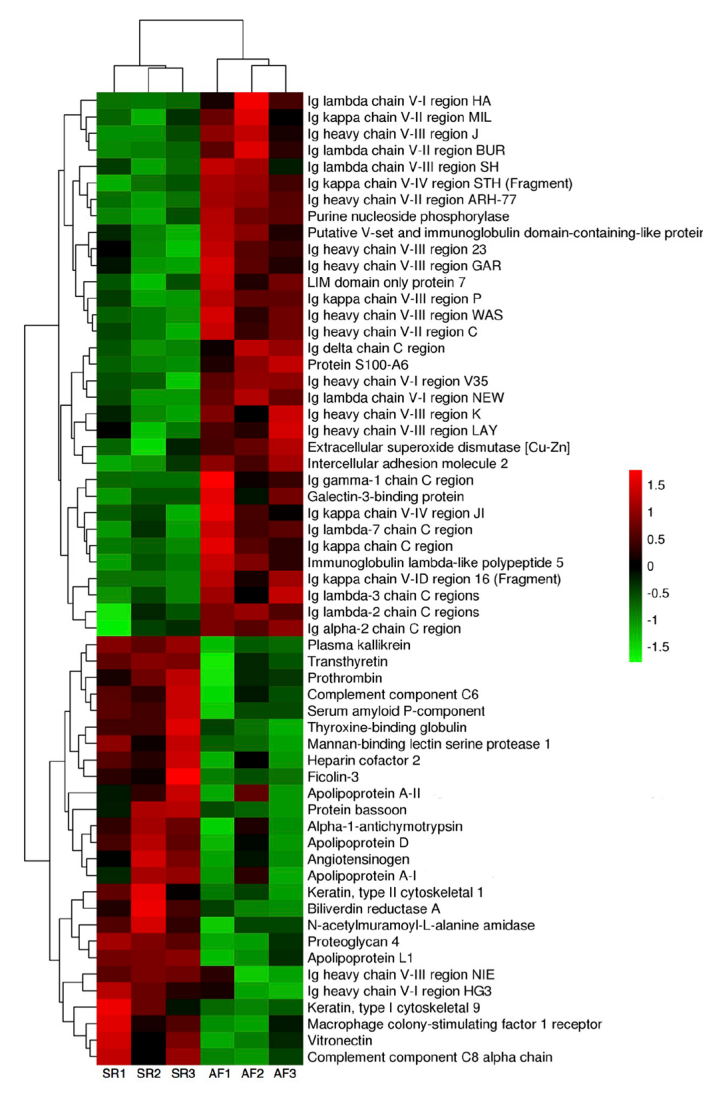

B

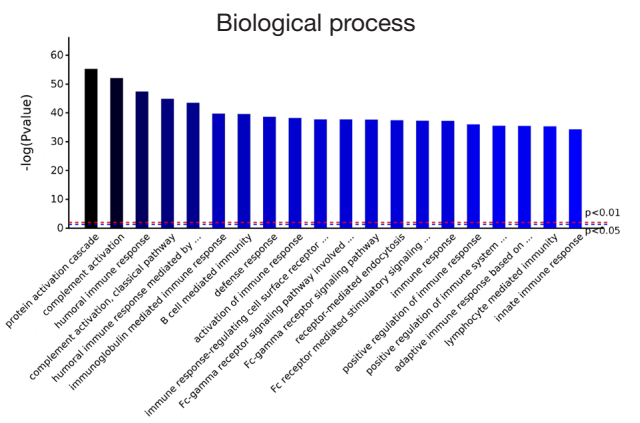

C
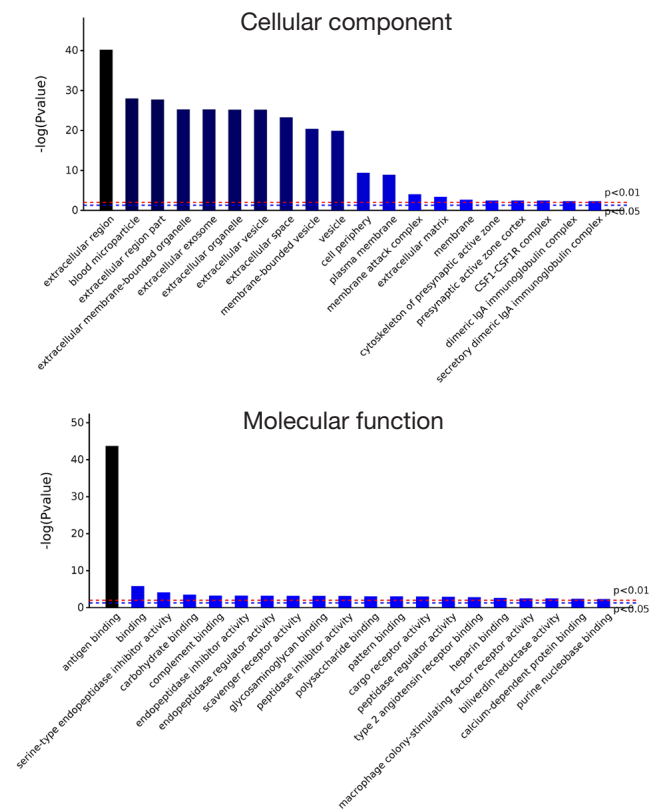

E

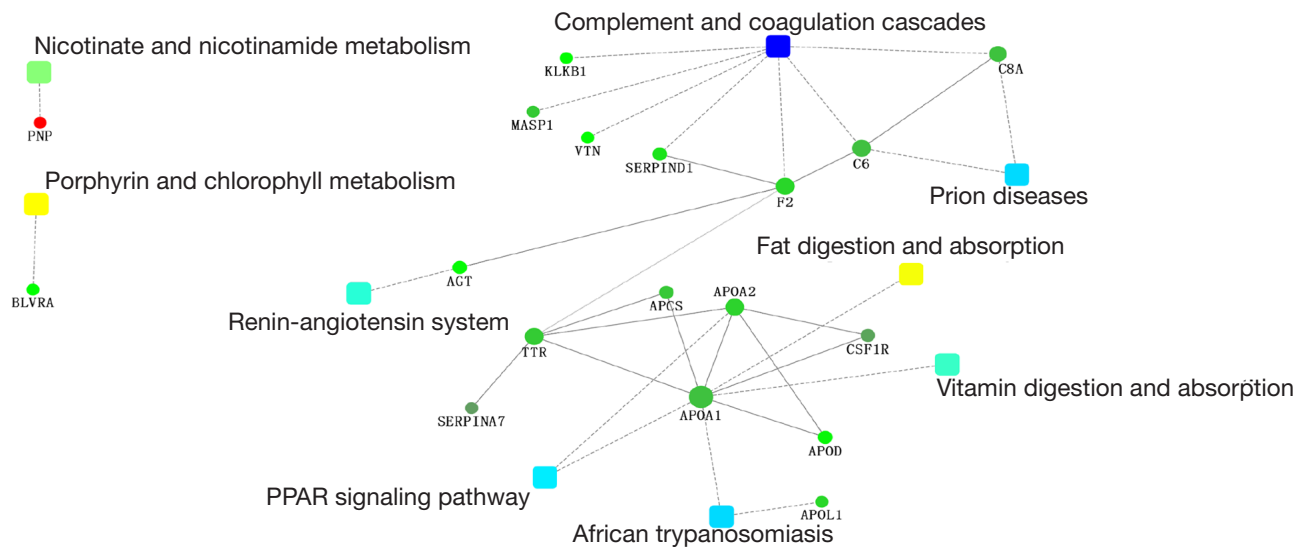

Figure 1 Analysis of proteomics in patients with AF compared to SR. (A) Unsupervised hierarchical clustering heatmap of proteins identified from the iTRAQ study (12 patients of AF and 12 patients of SR). The functional cluster analysis of DPs (left of heatmap) and the protein expression values (red: up-regulation, green: down-regulation) are demonstrated. (B,C,D) Classification of identified DPs based on their functional annotations using GO function and enrichment analysis: (B) biological process, (C) cell component, and (D) molecular function. (E) PPI network. Circular and square nodes represent the identified proteins and KEGG pathway terms, respectively. AF, atrial fibrillation; SR, sinus rhythm; iTRAQ, isobaric tags for relative and absolute quantitation; DPs, differential proteins; GO, Gene Ontology; PPI, protein-protein interaction; KEGG, Kyoto Encyclopedia of Genes and Genomes. 


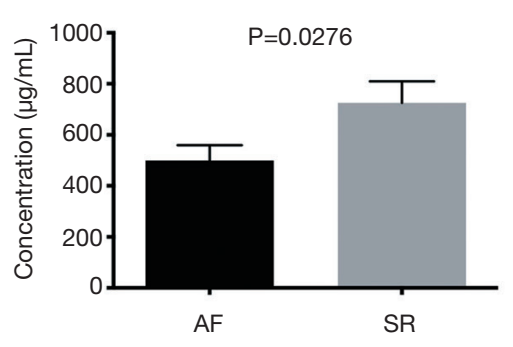

Apolipoprotein A-I

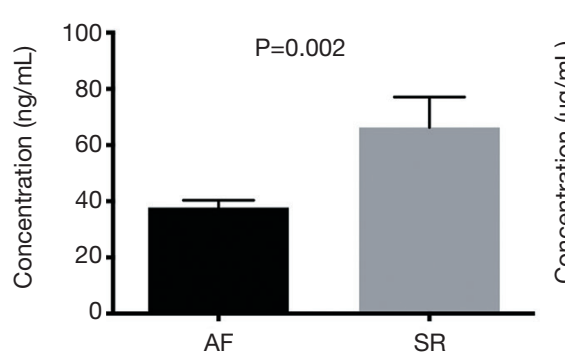

Macrophage colony-stimulating factor 1 receptor
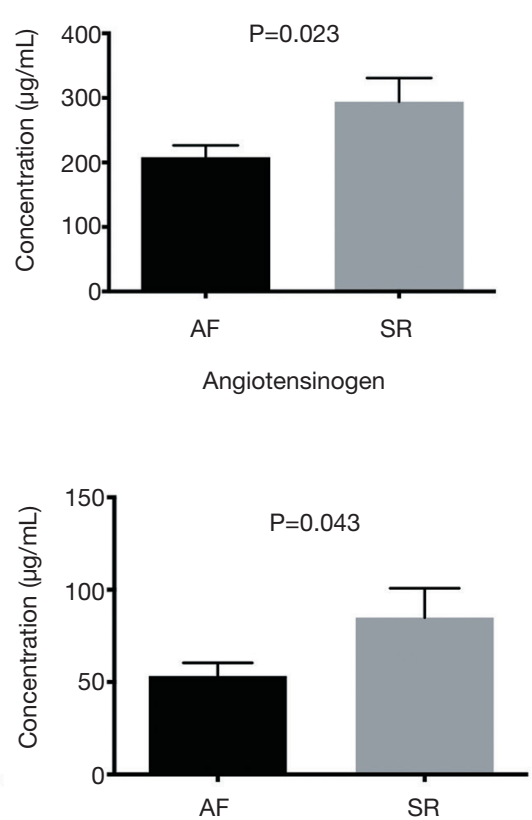

Vitronectin
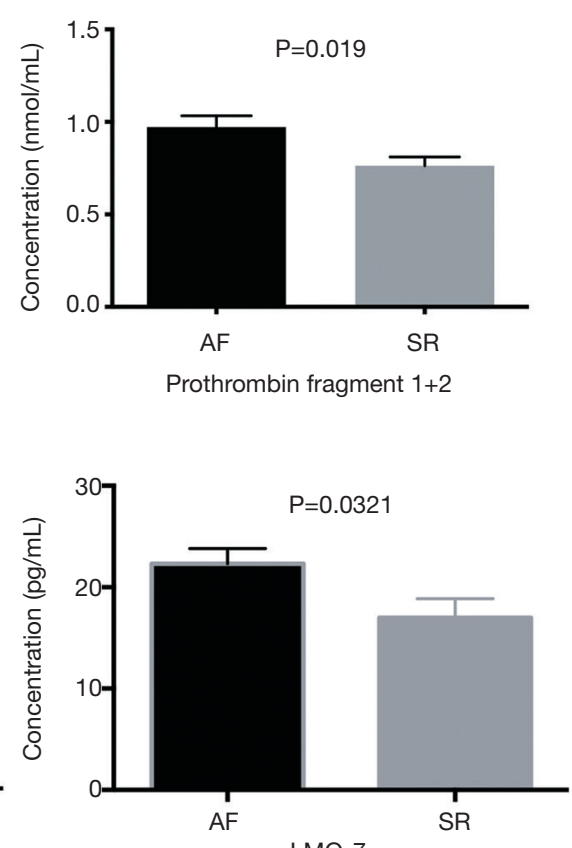

LMO-7

Figure 2 Validation of DPs identified from proteomics study. ELISA validation of DPs in AF patients ( $\mathrm{n}=50$ ) and SR patients ( $\mathrm{n}=30$ ). The figure shows that plasma levels of ApoA-I, angiotensinogen, CSF-1R, and VN were lower in AF patients compared to SR patients. Furthermore, the plasma level of LMO7 and prothrombin fragment 1+2 was higher in AF patients compared to SR patients. Data are shown as mean \pm SEM. DPs, differential proteins; ELISA, enzyme-linked immunosorbent assay; AF, atrial fibrillation; SR, sinus rhythm; ApoA-I, apolipoprotein A-I; CSF-1R, colony-stimulating factor 1 receptor; VN, vitronectin; LMO7, LIM domain only protein 7; SEM, standard error of the mean.

displayed in Figure 3. The sucrose concentration, cellobiose, lactose, raffinose, and galactinol was elevated in AF, along with several unsaturated fatty acids (such as arachidonic acid, palmitic acid, and linoleic acid). Furthermore, bioinformatics analysis was performed, and metabolic pathways including galactose metabolism, biosynthesis of unsaturated fatty acids, linoleic acid metabolism, ATPbinding cassette (ABC) transporters, and starch and sucrose metabolism were enriched, demonstrating a close connection between $\mathrm{AF}$ and these pathways.

\section{Combined proteomics and metabolomics analysis in chronic $A F$}

We integrated the proteomics and metabolomics datasets to generate a global view of AF patients' plasma signatures. The global cross-correlation analysis of identified metabolites and proteins demonstrated the recapitulation of known metabolome-proteome reaction models. As a result, the connection between identified proteins, metabolites, and several known enzymatic reaction models were established in Figure 4. On the other hand, KEGG mapping tools were used to integrate the above proteomics and metabolomics datasets into the human metabolic reference map (hsa01100) to provide a unified metabolic signature. As shown in Figure 4, each node represents a metabolite, the concentration of which was altered between the two groups. There were concurrent changes in both metabolites and proteins in the PPAR $\alpha$ pathway and nucleotide metabolism. Notably, ELISA validation of PNP, an enzyme that regulates several purine metabolism reactions, revealed that the concentration of PNP was not significantly different between the two groups. Interestingly, proteomics and metabolomics analyses revealed that two proteins in the PPAR $\alpha$ pathway, ApoA-I, and ApoA-II, were down-regulated in the AF patients.

\section{Discussion}

In the present combined study of proteomics and metabolomics on the plasma of patients with heart valve 

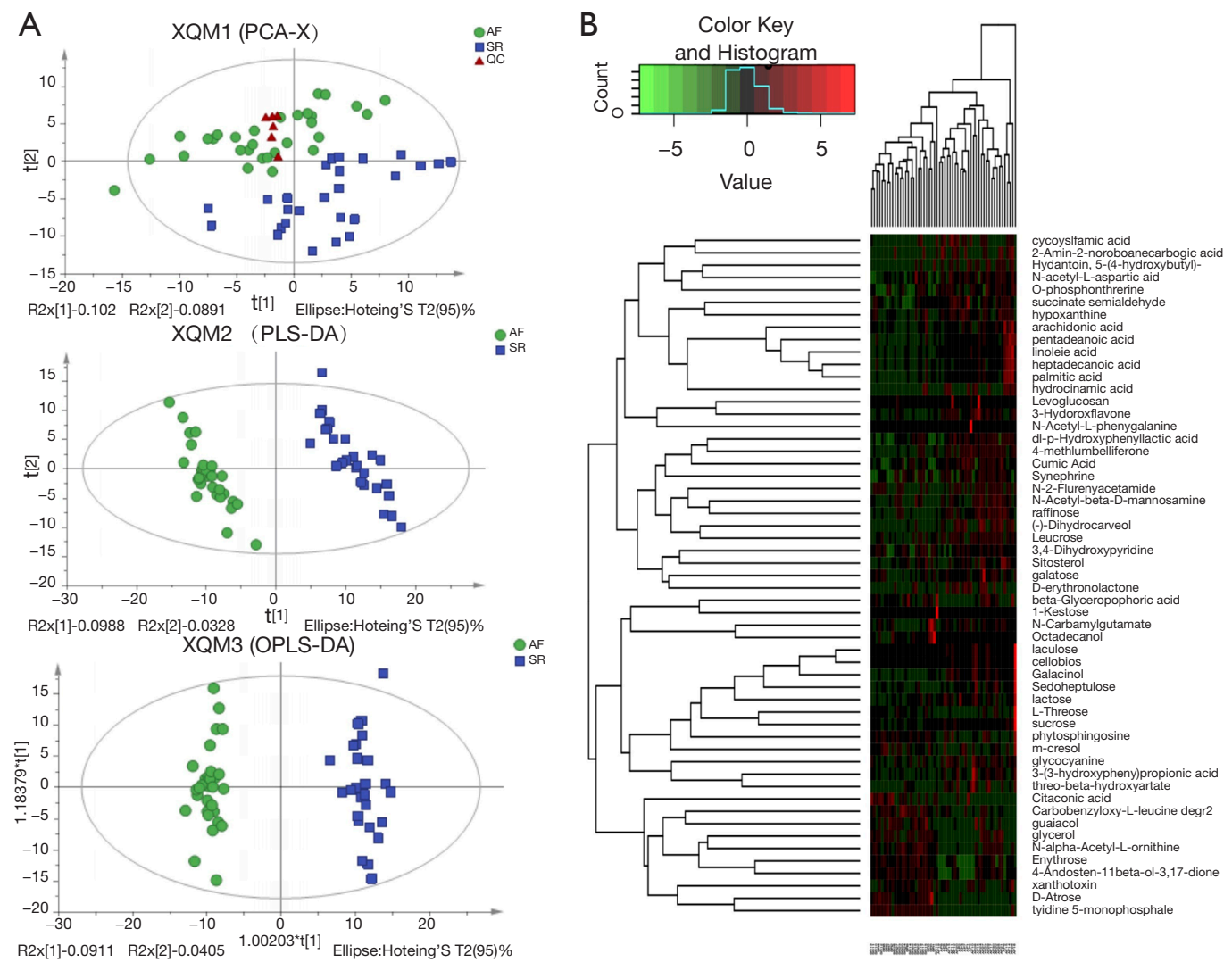

Figure 3 Metabolomics analysis in patients with AF compared to SR. Patients of AF ( $\mathrm{n}=30$ ) or SR (n=26) were analyzed by GC-MS. (A) PCA, PLS-DA, and OPLS-DA of the data in this study. PCA scores plot displays the variability among AF, SR, and QC samples; PLS-DA plot and OPLS-DA plot compare the variability between AF and SR samples. The quality of the models is described by the the proportion of variance in the data explained by the $\mathrm{X}$ and $\mathrm{Y}$ models and indicates goodness of fit (R2X and R2Y) values. (B) Heatmap of the metabolites in the GC-MS study. Fifty-five metabolites (rows) distinguished AF patients from SR patients (columns) under the condition of VIP $>1$ in the PLS-DA model and P values $<0.05$ in the Student's t-test. Unsupervised hierarchical clustering was performed using the R Programming Language (version 3.3.1). AF, atrial fibrillation; SR, sinus rhythm; GC-MS, gas chromatography-mass spectrometry; PCA, principal component analysis; PLS-DA, partial least-squares discriminant analysis; OPLS-DA, orthogonal partial least-squares discriminant analysis; QC, quality control; VIP, variable importance in projection.

disease, we found that: (I) among the 447 proteins and 322 metabolites detected, 57 proteins and 55 metabolites were differentially expressed between AF patients and SR patients; (II) by using bioinformatics tools, several pathways, particularly the PPAR $\alpha$ pathway, were enriched; (III) other enriched pathways included the metabolic pathway, renin angiotensin aldosterone system (RAAS), galactose metabolism, biosynthesis of unsaturated fatty acid, and linoleic acid metabolism; and (IV) several DPs and metabolites, such as ApoA-I, ApoA-II, and LMO7, may be further developed as biomarkers for AF in patients with MVD in order to monitor disease progress. The results from our integrated omics study in the plasma of MVD patients with AF compared to MVD patients in SR have not been previously reported (see Table S5 for published studies). Figure 5 is a schema that briefly demonstrates our findings. The findings of this study complement the results of other studies regarding AF $(18,19)$. However, our study provides important insights into multi-omics changes in MVD-associated AF.

In this study, down-regulation of ApoA-I was observed in the AF patients. In comparison, the change of ApoA-II was only found in the proteomics study. ApoA-I and ApoAII are the major components of high-density lipoprotein 


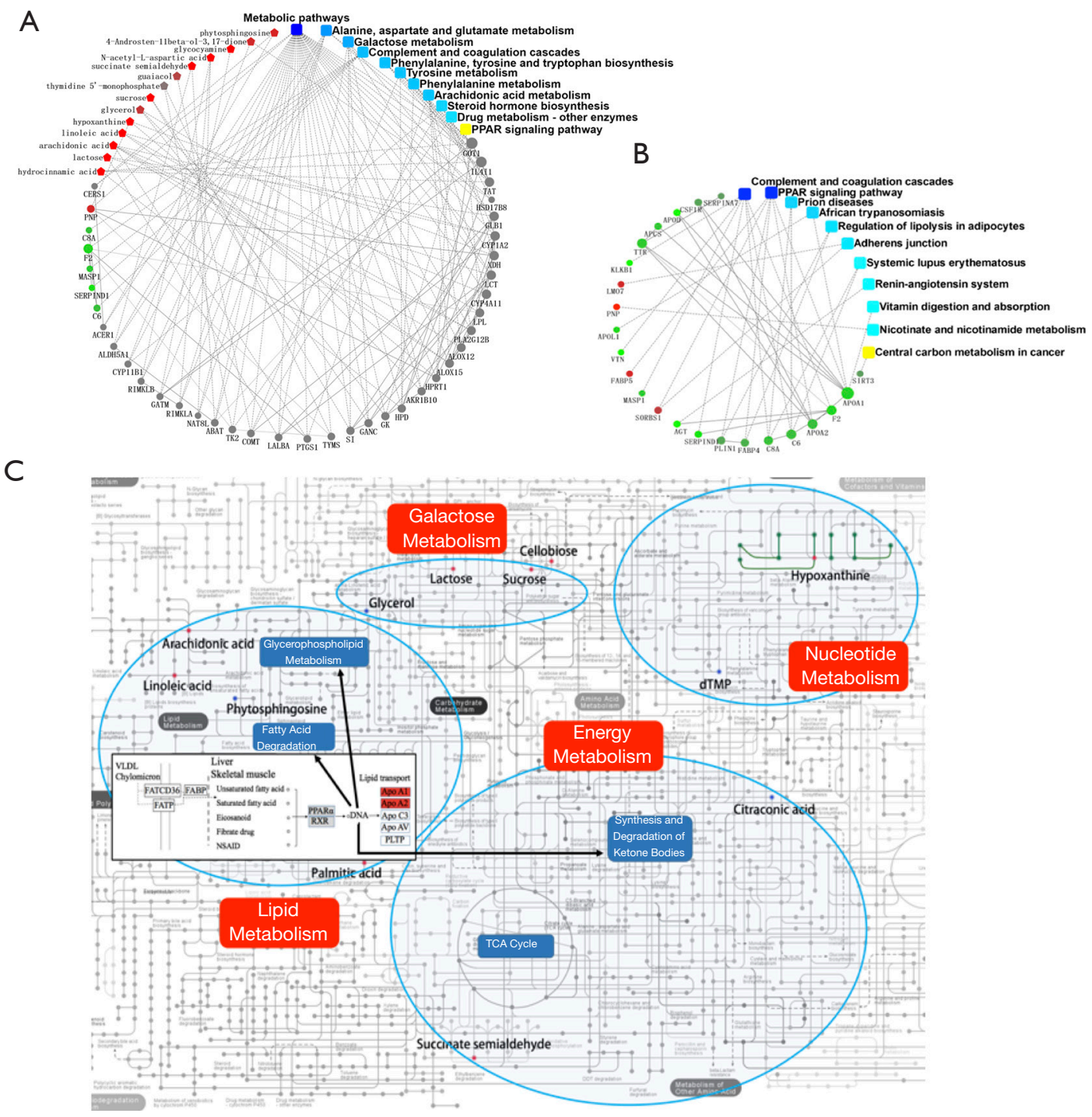

Figure 4 Integrated "-omics" analysis reveals global metabolic changes. (A) Cross-correlation analysis of identified proteins and metabolites from plasma samples. (B) PPI analysis of plasma proteomics findings. Note that PPAR pathway is now listed as one of the top pathways. Circular nodes represent the identified up- (red) and down-regulated (green) proteins, square nodes represent the KEGG pathway terms, and pentagonal nodes represent the identified metabolites. Solid or dashed grey lines between the molecules indicate the interaction with bigger and smaller confidence scores, respectively. (C) KEGG mapping tools were used to integrate the proteomics and metabolomics data sets into the human metabolic reference map (hsa01100). Red and blue represent up- and down-regulated, respectively. Lines represent reactions regulated by differentially expressed proteins and dots represent metabolites. Blue shading over lipid metabolism, galactose metabolism, nucleotide metabolism, and energy metabolism serve to highlight pathways that are highly represented. PPI, protein-protein interaction; PPAR, peroxisome proliferator-activated receptor; KEGG, Kyoto Encyclopedia of Genes and Genomes.

cholesterol (HDL-C), and the genes encoding these two proteins are activated by PPAR $\alpha$ via direct transcriptional control (20). PPAR is a major transcriptional regulator of fatty acid metabolism (21). The PPAR pathway regulates the expression of various genes, including proteins involved in fatty acid degradation, glycerophospholipid metabolism, synthesis and degradation of ketone bodies, etc. (16).

Moreover, PPAR $\alpha$ is a member of the ligand-activated nuclear receptor superfamily (22), and the dimer binds 


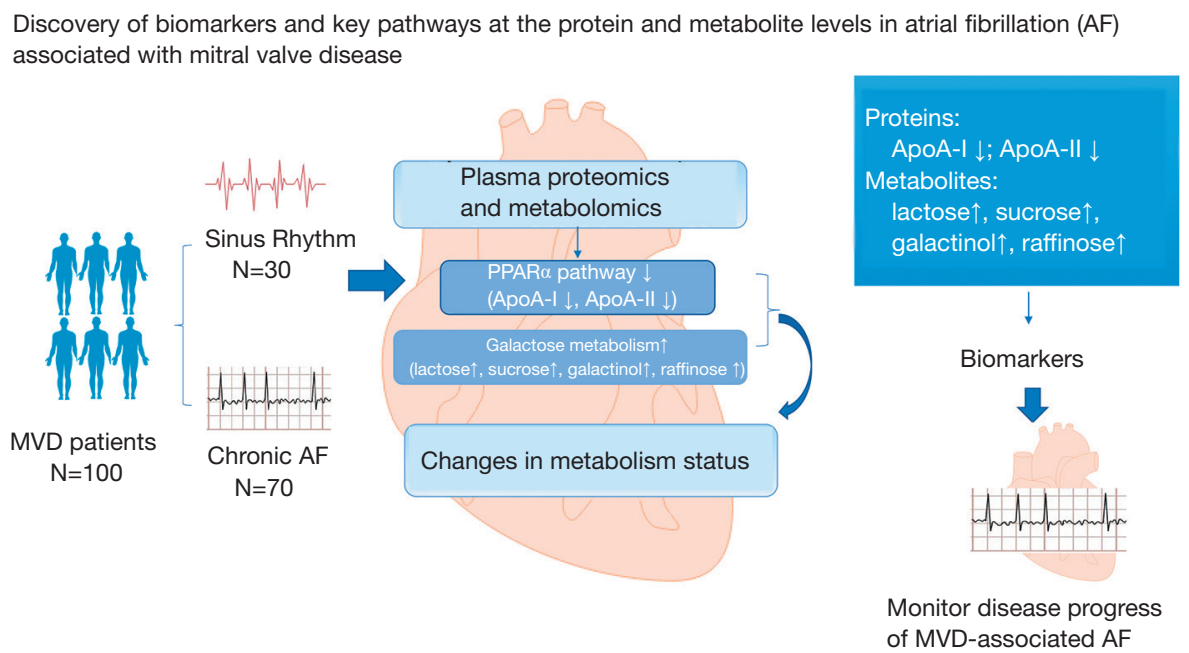

Figure 5 Schema of discovery of biomarkers and key pathways at the protein and metabolite levels in AF associated with MVD. The potential biomarkers and key pathways were investigated in the plasma of MVD patients with chronic AF by using multi-omics technologies. Dysregulation of the PPAR $\alpha$ pathway (lower ApoA-I and ApoA-II) and upregulation of the galactose metabolism (higher lactose, sucrose, galactinol, and raffinose) indicates changes in the metabolism status in patients with MVD-associated AF. These proteins/metabolites may be developed as biomarkers to monitor the disease progress of MVD-associated AF. AF, atrial fibrillation; MVD, mitral valve disease; PPAR $\alpha$, peroxisome proliferators-activated receptor alpha; ApoA-I, apolipoprotein A-I; ApoA-II, apolipoprotein A-II.

to PPAR response element (PPRE) on the promoter region of target genes and activates their expression (23). PPAR $\alpha$ also serves as a xenobiotic and lipid sensor to regulate fatty acid oxidation, fatty acid storage, lipid uptake, triacylglycerol (TAG) synthesis, glucose oxidation, lipoprotein synthesis, and inflammatory responses, and thus plays a key role in energy combustion (24). The down-regulation of PPAR $\alpha$ is most likely attributable to the down-regulation of ApoA-I and ApoA-II. As a result of PPAR $\alpha$ down-regulation, lipid metabolism is suppressed, and glucose oxidation is activated to force the heart to utilize more glucose instead of fatty acids. This notion is supported by the present study's metabolomic findings, as the concentration of plasma unsaturated fatty acids increased, indicating the accumulation of fatty acids in the circulation. Considering these findings, we hypothesize that AF is associated with the heart's altered metabolic status and $\operatorname{PPAR} \alpha$ plays an important role in this alternation (Figure 6). Our study suggests that AF may impair the heart's energy metabolism, and thus, the balance between lipid uptake and utilization is compromised. As a result, metabolic remodeling exists in the heart and is involved in the development of AF.

Several other proteins were also validated in the plasma of AF patients. Angiotensinogen is an essential component of the RAAS. It has recently been established that angiotensinogen and RAAS are closely associated with $\mathrm{AF}$ initiation and maintenance (25). It was reported that angiotensinogen gene polymorphisms and several other RAAS-related gene polymorphisms were associated with non-familial structural AF (26). The present study demonstrated that angiotensinogen concentration decreased in MVD patients with AF compared to those with SR. However, further exploration is required to understand the role of RAAS in AF better.

LMO7 is a member of the LIM domain-containing protein family and has been linked to the actin cytoskeleton organization. Interestingly, it has been reported that LMO7 is functionally involved in mammalian heart development, especially the development of the conduction system, and that knockdown of the LMO7 gene in zebrafish model may lead to arrhythmia, bradycardia, and delocalization of the heart (27). The present study showed up-regulation of LMO7 in the AF patients, suggesting that LMO7 plays a pathophysiological role in AF development. To our knowledge, this study is the first to reveal the alternation of the LMO7 protein in MVD-associated AF patients. These results suggest that the plasma level of LMO7 may be developed as a biomarker of AF.

$\mathrm{VN}$ is a glycoprotein found in serum and tissues, and 


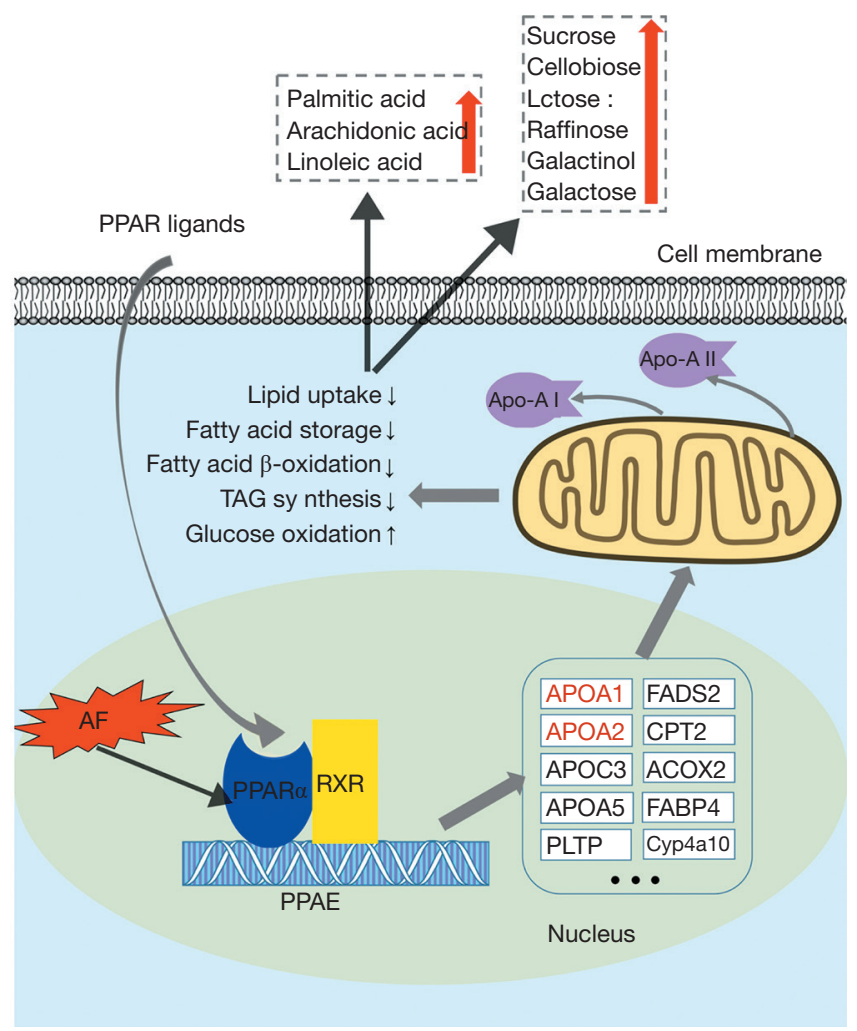

Figure 6 PPAR $\alpha$ plays a crucial role in the lipid metabolism in the process of AF. PPAR $\alpha$ heterodimerizes in the nucleus with RXR, and then the dimer binds to the PPRE on the promoter region of target genes to activate their expression. Down-regulation of PPAR in the process of AF inhibits the expression of PPAR $\alpha$ targeted genes, resulting in down-regulation of lipid uptake, fatty acid storage, fatty acid $\beta$-oxidation and TAG synthesis, as well as up-regulation of glucose oxidation. Therefore, the concentration of circulating unsaturated fatty acids (palmitic acid, arachidonic acid, linoleic acid) is increased due to decreased consumption, while the concentration of carbohydrates (sucrose, cellobiose, lactose, et al.) is increased due to complexed compensate mechanisms to fulfill the energy demands of the heart. PPAR $\alpha$, peroxisome proliferators-activated receptor alpha; AF, atrial fibrillation; RXR, retinoid X receptors; PPRE, PPAR response element; TAG, tricarboxylic acid cycle.

serves as a cell-to-substrate adhesion molecule. Biological processes, including immune response, complement activation, and vascular endothelial growth factor receptor signaling pathway regulation, are also related to $\mathrm{VN}$ (28). $\mathrm{VN}$ inhibits the membrane-damaging effect of the terminal cytolytic complement pathway (28). In this study, we observed a down-regulation of $\mathrm{VN}$ concentration in $\mathrm{AF}$ patients, suggesting that the complement system is activated in MVD-associated AF, and that the plasma level of $\mathrm{VN}$ may be a potential biomarker of AF.

In this study, AF's development was inevitably correlated to some clinical variables, such as left atrium (LA) size (see Tables S1,S2). Nevertheless, our study aimed to identify biomarkers of AF. Therefore, we included all patients who satisfied the study's selection criteria and divided them into two groups according to the heart rhythm (either sinus or AF). The differences in the protein and metabolite levels between the two groups were then investigated. The above DPs and metabolites were successfully identified as potential biomarkers for $\mathrm{AF}$.

\section{Study limitations}

This study has some limitations that should be noted. Both proteomics and metabolomics studies reveal abundant unnamed proteins and metabolites. In order to perform bioinformatics analyses, the corresponding identification (ID) in certain databases is required. In fact, some proteins or metabolites failed to be recognized by these databases, and therefore the data were "invalid" and were unable to be 
analyzed at this stage. With future advances in technology, more information may be provided.

\section{Conclusions}

In conclusion, the present study, for the first time, identified a large number of DPs and metabolites by using multi-omics technologies in MVD-associated AF patients. Our study provides an integrative view with the combined analyses of proteomics and metabolomics, and reveals that the PPAR signaling pathway, and particularly PPAR $\alpha$ pathway, plays an important role in AF. Other pathways involved in MVD-associated AF are the RAAS, galactose metabolism, biosynthesis of unsaturated fatty acids, and linoleic acid metabolism. Some identified DPs and metabolites, such as ApoA-I, ApoA-II, LMO7, and $\mathrm{VN}$, may be further developed as biomarkers for MVDassociated $\mathrm{AF}$ patients to monitor disease progress.

\section{Acknowledgments}

We acknowledge the surgeons and the head nurse (KaiJing Wei) and her nursing staff for assistance in collecting samples.

Funding: This work was supported by the National Natural Science Foundation of China (81870288 \& 81641017); the Non-profit Central Research Institute Fund of Chinese Academy of Medical Sciences (2017NL31001 \& 2018TX31002 \& 2019XK310001\& 2020-PT310-007); and the Tianjin Science and Technology Commission (18PTZWHZ00060).

\section{Footnote}

Reporting Checklist: The authors have completed the STROBE reporting checklist. Available at http://dx.doi. org/10.21037/atm-20-3767

Data Sharing Statement: Available at http://dx.doi. org/10.21037/atm-20-3767

Conflicts of Interest: All authors have completed the ICMJE uniform disclosure form (available at http://dx.doi. org/10.21037/atm-20-3767). All authors report that they have a patent pending for a diagnostic kit of multiple protein biomarkers and its application in atrial fibrillation. The authors have no other conflicts of interest to declare. Ethical Statement: The authors are accountable for all aspects of the work in ensuring that questions related to the accuracy or integrity of any part of the work are appropriately investigated and resolved. This study was approved by the Ethics Committee of the TEDA International Cardiovascular Hospital \{No. [2019]-0315-1\} and conforms to the principles outlined in the Declaration of Helsinki (as revised in 2013). Written informed consent was obtained from each patient before being included in the study.

Open Access Statement: This is an Open Access article distributed in accordance with the Creative Commons Attribution-NonCommercial-NoDerivs 4.0 International License (CC BY-NC-ND 4.0), which permits the noncommercial replication and distribution of the article with the strict proviso that no changes or edits are made and the original work is properly cited (including links to both the formal publication through the relevant DOI and the license). See: https://creativecommons.org/licenses/by-nc-nd/4.0/.

\section{References}

1. Chugh SS, Havmoeller R, Narayanan K, et al. Worldwide epidemiology of atrial fibrillation: a Global Burden of Disease 2010 Study. Circulation 2014;129:837-47.

2. Fan K, Lee KL, Chiu CS, et al. Effects of biatrial pacing in prevention of postoperative atrial fibrillation after coronary artery bypass surgery. Circulation 2000;102:755-60.

3. Andrade J, Khairy P, Dobrev D, et al. The clinical profile and pathophysiology of atrial fibrillation: relationships among clinical features, epidemiology, and mechanisms. Circ Res 2014;114:1453-68.

4. Vinereanu D, Wang A, Mulder H, et al. Outcomes in anticoagulated patients with atrial fibrillation and with mitral or aortic valve disease. Heart 2018;104:1292-9.

5. Gao G, Xuan C, Yang Q, et al. Identification of altered plasma proteins by proteomic study in valve heart diseases and the potential clinical significance. PLoS One 2013;8:e72111.

6. Jiang YY, Hou HT, Yang Q, et al. Chloride channels are involved in the development of atrial fibrillation - a transcriptomic and proteomic study. Sci Rep 2017;7:10215.

7. Liu H, Chen G, Zheng H, et al. Differences in atrial fibrillation associated proteins between the left and right atrial appendages from patients with rheumatic mitral valve disease: a comparative proteomic analysis. Mol Med Rep 2016;14:4232-42.

8. Mayr M, Yusuf S, Weir G, et al. Combined metabolomic 
and proteomic analysis of human atrial fibrillation. J Am Coll Cardiol 2008;51:585-94.

9. Chen HX, Li MY, Jiang YY, et al. Role of PPAR pathway in atrial fibrillation associated with heart valvular disease: transcriptomics and proteomics in the human atrial tissue. Signal Transduct Target Ther 2020;5:4.

10. Li XY, Hou HT, Chen HX, et al. Preoperative plasma biomarkers associated with atrial fibrillation after coronary artery bypass surgery. J Thorac Cardiovasc Surg 2020. [Epub ahead of print].

11. He GW, Hou HT, Xuan C, et al. Corrective surgery alters plasma protein profiling in congenital heart diseases and clinical perspectives. Am J Transl Res 2020;12:1319-37.

12. Xuan C, Gao G, Yang Q, et al. Proteomic study reveals plasma protein changes in congenital heart diseases. Ann Thorac Surg 2014;97:1414-9.

13. Zhang X, Hou HT, Wang J, et al. Plasma proteomic study in pulmonary arterial hypertension associated with congenital heart diseases. Sci Rep 2016;6:36541.

14. Zhang X, Wang K, Yang Q, et al. Acute phase proteins altered in the plasma of patients with congenital ventricular septal defect. Proteomics Clin Appl 2015;9:1087-96.

15. Guo ZP, Hou HT, Jing R, et al. Plasma protein profiling in patients undergoing coronary artery bypass grafting surgery and clinical significance. Oncotarget 2017;8:60528-38.

16. Pierce A, Unwin RD, Evans CA, et al. Eight-channel iTRAQ enables comparison of the activity of six leukemogenic tyrosine kinases. Mol Cell Proteomics 2008;7:853-63.

17. Kanehisa M, Furumichi M, Tanabe M, et al. KEGG: new perspectives on genomes, pathways, diseases and drugs. Nucleic Acids Res 2017;45:D353-61.

18. Wu J, Corr PB. Influence of long-chain acylcarnitines on voltage-dependent calcium current in adult ventricular myocytes. Am J Physiol 1992;263:H410-7.

19. Fabritz L, Guasch E, Antoniades C, et al. Expert consensus document: Defining the major health modifiers causing atrial fibrillation: a roadmap to underpin

Cite this article as: Li MY, Chen HX, Hou HT, Wang J, Liu XC, Yang Q, He GW. Biomarkers and key pathways in atrial fibrillation associated with mitral valve disease identified by multi-omics study. Ann Transl Med 2021;9(5):393. doi: 10.21037/atm-20-3767 personalized prevention and treatment. Nat Rev Cardiol 2016;13:230-7.

20. Lefebvre P, Chinetti G, Fruchart JC, et al. Sorting out the roles of PPAR alpha in energy metabolism and vascular homeostasis. J Clin Invest 2006;116:571-80.

21. Khera AV, Millar JS, Ruotolo G, et al. Potent peroxisome proliferator-activated receptor-? agonist treatment increases cholesterol efflux capacity in humans with the metabolic syndrome. Eur Heart J 2015;36:3020-2.

22. Kliewer SA, Umesono K, Noonan DJ, et al. Convergence of 9-cis retinoic acid and peroxisome proliferator signalling pathways through heterodimer formation of their receptors. Nature 1992;358:771-4.

23. Bishop-Bailey D. Peroxisome proliferator-activated receptors in the cardiovascular system. Br J Pharmacol 2000;129:823-34.

24. Mukherjee R, Locke KT, Miao B, et al. Novel peroxisome proliferator-activated receptor alpha agonists lower lowdensity lipoprotein and triglycerides, raise high-density lipoprotein, and synergistically increase cholesterol excretion with a liver $\mathrm{X}$ receptor agonist. J Pharmacol Exp Ther 2008;327:716-26.

25. Kirchhof P, Fabritz L. Of hammers and screws: reninangiotensin-aldosterone system inhibition to prevent atrial fibrillation in patients with hypertension. Eur Heart J 2014;35:1169-71.

26. Tsai CT, Lai LP, Lin JL, et al. Renin-angiotensin system gene polymorphisms and atrial fibrillation. Circulation 2004;109:1640-6.

27. Ott EB, van den Akker NM, Sakalis PA, et al. The LMO7 is important in zebrafish heart development. Dev Dyn 2008;237:3940-52.

28. Wang Y, Liu XC, Zhang GW, et al. A new transmyocardial degradable stent combined with growth factor, heparin, and stem cells in acute myocardial infarction. Cardiovasc Res 2009;84:461-9.

(English Language Editor: A. Kassem) 


\section{Materials and methods}

\section{Patient recruitment and sample collection}

One hundred patients with HVD who came to TEDAInternational Cardiovascular Hospital, Tianjin, China, for surgery from January 2013 to December 2017 were enrolled in this study. All patients underwent mitral valve replacement. Patients with chronic AF or SR were assigned to the AF group $(n=70)$ or SR group $(n=30)$, respectively. A diagnosis of AF was made based on the medical history, physical examination, and a 12-lead electrocardiogram before and after admission for the valve surgery. For comparison, patients in SR were screened to ensure that they had no AF by direct questioning about symptoms suggestive of $\mathrm{AF}$ and by the analysis of all 12lead electrocardiograms before and after admission for valve surgery. Patients with infective endocarditis, sick sinus syndrome, familial paroxysmal AF (lone AF/familial AF), pulmonary heart disease, cardiomyopathy, hyperthyroidism, and chronic kidney diseases were excluded from this study. Patients who were diagnosed with coronary atherosclerotic heart disease or under the age of 18 were also excluded. The demographic characteristics of all patients enrolled in this study are shown in Table S1.

The study was approved by the Ethics Committee of TEDA-international cardiovascular hospital and written informed consent was obtained from all patients enrolled in the study.

Blood samples were collected in EDTA tubes from patients the day before surgery and the plasma was then centrifuged at 3,500 rpm for $10 \mathrm{~min}$ to separate from blood cells.

\section{Proteomics analysis}

A discovery-validation workflow was used in the proteomics analysis. iTRAQ technology was used for the proteomics study. Because there are eight Isobaric tags ( $\mathrm{m} / \mathrm{z} 113,114$, $115,116,117,118,119$ and 121 ), the plasma samples from 24 patients were divided into six groups (three AF group and three SR group), each of these groups contained the plasma from four patients. In addition, plasma samples from $12 \mathrm{AF}$ or $12 \mathrm{SR}$ patients were used as control groups, respectively (Figure S1). Thus, the eight Isobaric tags were concomitantly labeled in the plasma samples for the iTRAQ study.

In the validation phase, identified DPs were validated in a larger study population using ELISA in plasma samples.

\section{Plasma bigh abundance protein depletion, protein extraction and quantification}

The ProteoExtractTM Albumin/IgG Removal Kit (CALBIOCHEM, USA) was used to remove more than $80 \%$ of the top-two (serum albumin and $\mathrm{IgG}$ ) high abundance proteins from plasma samples according to manufacturer's instructions. Total protein from sample was extracted by ProteoPrep ${ }^{\circledR}$ Total Extraction Sample Kit (Sigma-Aldrich, USA). The eluted samples were mixed in 5 volumes of cold acetone and stored at $-20^{\circ} \mathrm{C}$ for 1 hour, followed by centrifugation at $5,000 \mathrm{~g}$ for 30 minutes. The deposit was dried and dissolved in lysis solution for 1 hour and the solution was again centrifuged at $15,000 \mathrm{~g}$ for 15 minutes. The supernatant was collected and Bradford method was used to determine the concentration of the protein extracts.

\section{Protein digestion and iTRAQ labeling}

Protein $(100 \mu \mathrm{g})$ from each sample was precipitated by cold acetone at $-20^{\circ} \mathrm{C}$ for 1 hour and then centrifuged at 12,000 rpm for 15 minutes, after which the deposit was collected and dissolved with $50 \mu \mathrm{L}$ dissolution buffer. Proteins in the solution were reduced $\left(4 \mu \mathrm{L}\right.$ reducing reagent, $60{ }^{\circ} \mathrm{C}$ for 1 hour), alkylated ( $2 \mu \mathrm{L}$ cycsteine-blocking reagents, room temperature for 10 minutes), digested [50 $\mu \mathrm{L}$ sequencing-grade trypsin $(50 \mathrm{ng} / \mu \mathrm{L}), 37^{\circ} \mathrm{C}$ for 12 hours] and centrifuged at 12,000 rpm for 20 minutes. Following digestion with trypsin, iTRAQ regent (AB SCIEX, Framingham, MA, USA) was added and each sample was labeled with a unique iTRAQ $\operatorname{tag}(\mathrm{m} / \mathrm{z} 113,114,115,116$, $117,118,119$ and 121).

\section{D-LC-MS/MS analysis}

The peptides were dried in a vacuum freeze dryer and resuspended in $100 \mu \mathrm{L}$ of buffer A. Reversed-phase liquid chromatography (RPLC) was used to fractionate sample on the Agilent 1200 HPLC system (Agilent, USA) equipped with HPLC column (Narrow-Bore $2.1 \times 150 \mathrm{~mm}, 5 \mu \mathrm{m}$, Agilent, USA). A total of 10 segments were collected at a rate of $0.3 \mathrm{~mL} / \mathrm{min}$ using a nonlinear binary gradient starting with buffer A and transitioning to buffer B. The fractions were dried in a vacuum freeze dryer and redissolved in nano-RPLC buffer A. Each segment was loaded on a Eksigent nanoLC-UltraTM 2D System (AB SCIEX, USA) mounted with a C18 nanoLC trap column $(100 \mu \mathrm{m}$ 
$\times 3 \mathrm{~cm}, \mathrm{C} 18,3 \mu \mathrm{m}, 150 \AA$ ) and separated over a $70 \mathrm{~min}$ acetonitrile gradient from $5 \%$ to $35 \%$ in $0.1 \%$ formic acid combined with a Triple TOF 5600 System (AB SCIEX, USA) fitted with a Nanospray III source (AB SCIEX, USA). Data were acquired using an ion spray voltage of $2.5 \mathrm{kV}$, curtain gas of 30PSI, nebulizer gas of 5PSI, and an interface heater temperature of $150^{\circ} \mathrm{C}$.

\section{iTRAQ data analysis and bioinformatics analysis}

After data pre-processing, standardization, and quality control, the original data were screened for reliable peptides and proteins. ID of peptides from tandem mass spectrometry (MS) was achieved using the Protein Pilot Software v.5.0 (AB SCIEX, USA) against Uniprot_Homo Sapiens Database to match the theory data in order to obtain result of protein ID. The criteria for DPs were established as: (I) unique peptide $\geq 1$, apart from invalid values and anti-library data; (II) FC $>1.2$ or $<0.83$; and (III) $\mathrm{P}$ value $<0.05$ ( $\mathrm{t}$-test for repeated data for over three times). Proteins fulfilling these criteria were considered to have significant difference in expression between the two groups. A list of DPs identified was implemented on the on-line software DAVID Bioinformatics Resources 6.8 (NIH) for GO (including biological process, molecular function and cellular component) function and enrichment analysis. KEGG pathway database (18) and STRING database were used to perform pathway analysis and PPI analysis.

\section{ELISA}

Following the success of the iTRAQ labeling and 2D-LCMS/MS screening for changes in protein abundance, ELISA was employed to validate the results of the proteomics analysis. Selection of candidate proteins for ELISA validation was based on (I) expressed differentially in AF patient group and SR patient group; (II) potential functional or pathological significance in AF; (III) more than 1 peptide was identified by LC-MS/MS; and (IV) not been reported before in $\mathrm{AF}$ patients at the protein level.

In new group of patients, the further validation of candidate proteins was performed by using human ELISA kits (CUSABIO Biotech Co., Ltd., Wuhan, China). By the selection criteria mentioned above, there were two proteins, carbamoyl-phosphate synthetase I and complement factor $\mathrm{H}$-related protein 2, that were further validated in the plasma. The methods followed the manufacturer's instructions.

\section{Metabolomics analysis}

Plasma samples were thawed at room temperature and $50 \mu \mathrm{L}$ of the sample was mixed with $10 \mu \mathrm{L}$ of internal standard (2-chloro-l-phenylalanine, $0.3 \mathrm{mg} / \mathrm{mL}$, dissolved in methanol) and then vortexed for $10 \mathrm{~s}$. Subsequently, $150 \mu \mathrm{L}$ of ice-cold mixture of methanol and acetonitrile $(2 / 1, \mathrm{vol} / \mathrm{vol})$ were added and then vortexed for $1 \mathrm{~min}$ and ultrasonicated for $5 \mathrm{~min}$. The sample was then placed at $-20{ }^{\circ} \mathrm{C}$ for $10 \mathrm{~min}$ and centrifuged at $15,000 \mathrm{rpm}$ for $10 \mathrm{~min}$. The samples were then prepared for GC-MS analysis.

\section{GC-MS analysis}

The samples destined for GC/MS analysis were dried in a freeze concentration centrifugal dryer and mixed with $80 \mu \mathrm{L}$ of $15 \mathrm{mg} / \mathrm{mL}$ methoxylamine hydrochloride in pyridine prior to being derivatized using BSTFA. After vortex (2 min) and incubation $\left(37^{\circ} \mathrm{C}, 90 \mathrm{~min}\right), 80 \mu \mathrm{L}$ of BSTFA (with $1 \%$ TMCS) and $20 \mu \mathrm{L}$-hexane were added to each sample and the mixture was again vortexed $(2 \mathrm{~min})$ and then derivatized $\left(70{ }^{\circ} \mathrm{C}, 60 \mathrm{~min}\right)$. A mixture of aliquots of all samples were used as QC controls and they were injected at regular intervals (every ten samples) throughout the analytical run. Samples were analyzed on an Agilent $7890 \mathrm{~B}$ gas chromatography system coupled to an Agilent 5977A MSD system (Agilent, CA, USA). Samples were injected $\left(1 \mu \mathrm{L}\right.$, splitless mode, $\left.260{ }^{\circ} \mathrm{C}\right)$ onto a DB-5MS fusedsilica capillary column $(30 \mathrm{~m} \times 0.25 \mathrm{~mm} \times 0.25 \mu \mathrm{m}$, Agilent J \& W Scientific, Folsom, CA, USA) and eluted $\left(1.0 \mathrm{~mL} / \mathrm{min} \mathrm{He}\right.$, initial temperature $50^{\circ} \mathrm{C}, 15^{\circ} \mathrm{C} / \mathrm{min}$ to $125^{\circ} \mathrm{C}$, $5^{\circ} \mathrm{C} / \mathrm{min}$ to $210^{\circ} \mathrm{C}, 10^{\circ} \mathrm{C} / \mathrm{min}$ to $270{ }^{\circ} \mathrm{C}, 20^{\circ} \mathrm{C} / \mathrm{min}$ to $305^{\circ} \mathrm{C}$, $5 \mathrm{~min}$ at $305^{\circ} \mathrm{C}$ ), ionized (EI, $70 \mathrm{eV}$ ), and scanned from 50 $450 \mathrm{~m} / \mathrm{z}$ after a solvent delay of $5 \mathrm{~min}$ (source $230^{\circ} \mathrm{C}$, quad at $150^{\circ} \mathrm{C}$ ). Data was analyzed using ChromaTOF software (v4.34, LECO, St. Joseph, MI, USA).

\section{Data processing and bioinformatics analysis}

ChromaTOF software and Fiehn database were used to process the raw data and provide qualitative information about the metabolites. Data were normalized by total peak area of each sample using Excel 2007 and imported into SIMCA-P software (version 14.0, Umetrics, Umeå, Sweden) for multivariate statistical analysis. Principal component analysis (PCA) was used to visualize the general distribution of samples and the stability of the whole analysis process. PLS-DA and orthogonal PLS-DA (OPLS-DA) were 
used to distinguish the differences of general metabolic profiling between different patient groups. To avoid model overfitting, a default of seven rounds of cross-validation in SIMCA-P software was applied. Moreover, OPLS-DA models were validated by a permutation analysis with 200 times. DMs were selected according to VIP values $>1$ in OPLS-DA model as well as $\mathrm{P}$ values $<0.05$ in Student's test (Figure S1). KEGG pathway database was used to generate significant pathways in metabolomics dataset.

\section{Creation of pathway maps}

All the omics data including transcriptomics, proteomics, and metabolomics data were used to compare proteins/ enzymes with metabolic pathways. Quantitative changes of individual metabolites and proteins as compared with control samples were calculated and graphed by using the KEGG mapping tools. In particular, by combining proteomics with metabolomics data, we were able to identify the common pathways involved in AF.

\section{Statistical analysis}

SPSS 23.0 software (SPSS Inc., Chicago, IL, USA) and GraphPad 6.0c software (GraphPad Software, La Jolla, CA, USA) were used for statistical analysis. Data are expressed as mean \pm SEM. Comparisons of statistical data were performed using either the independent samples $t$-test or Chi-square tests. A P value of $<0.05$ was considered statistically significant. 
A

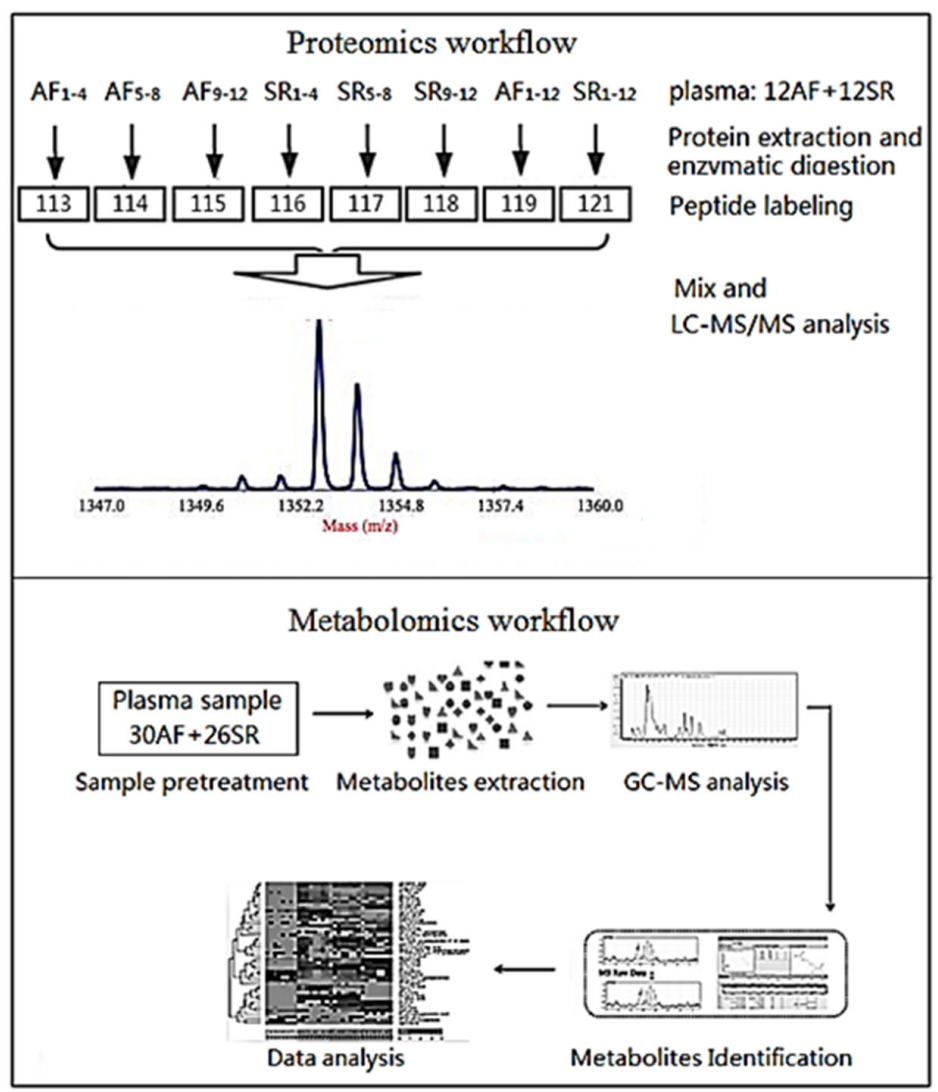

B

Proteomic Study

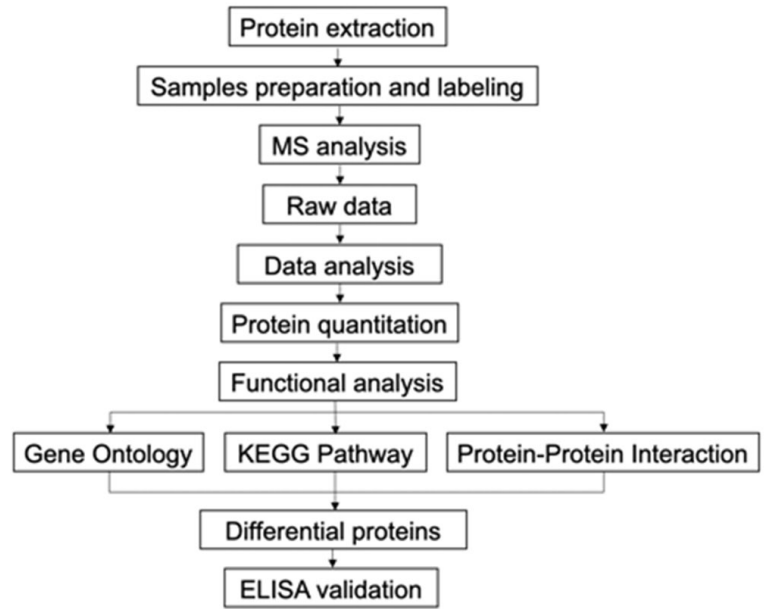

Metabolomic Study

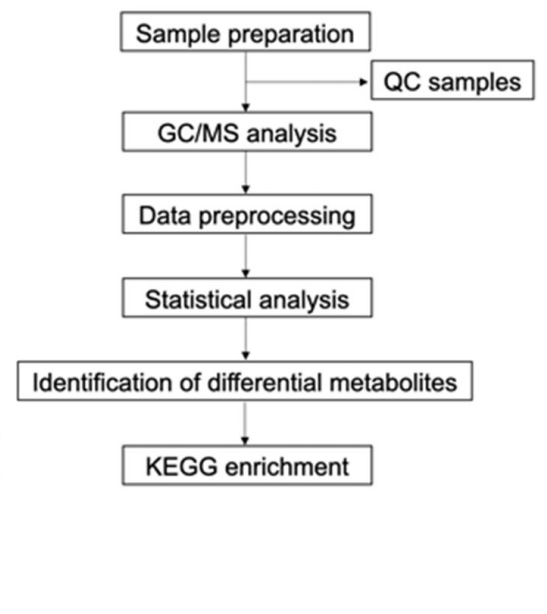

Figure S1 Proteomics (iTRAQ) and metabolomics workflow of plasma samples in the study. (A) Workflow of proteomics (iTRAQ) and metabolomics in plasma samples. AF, atrial fibrillation samples; SR, sinus rhythm samples; AF1-12, plasma from AF patients; SR1-12, plasma from SR patients. (B) Flowchart of the proteomics (iTRAQ) and metabolomics procedures. iTRAQ, isobaric tags for relative and absolute quantitation. 
Table S1 Demographics of study population in the study of iTRAQ and GC-MS

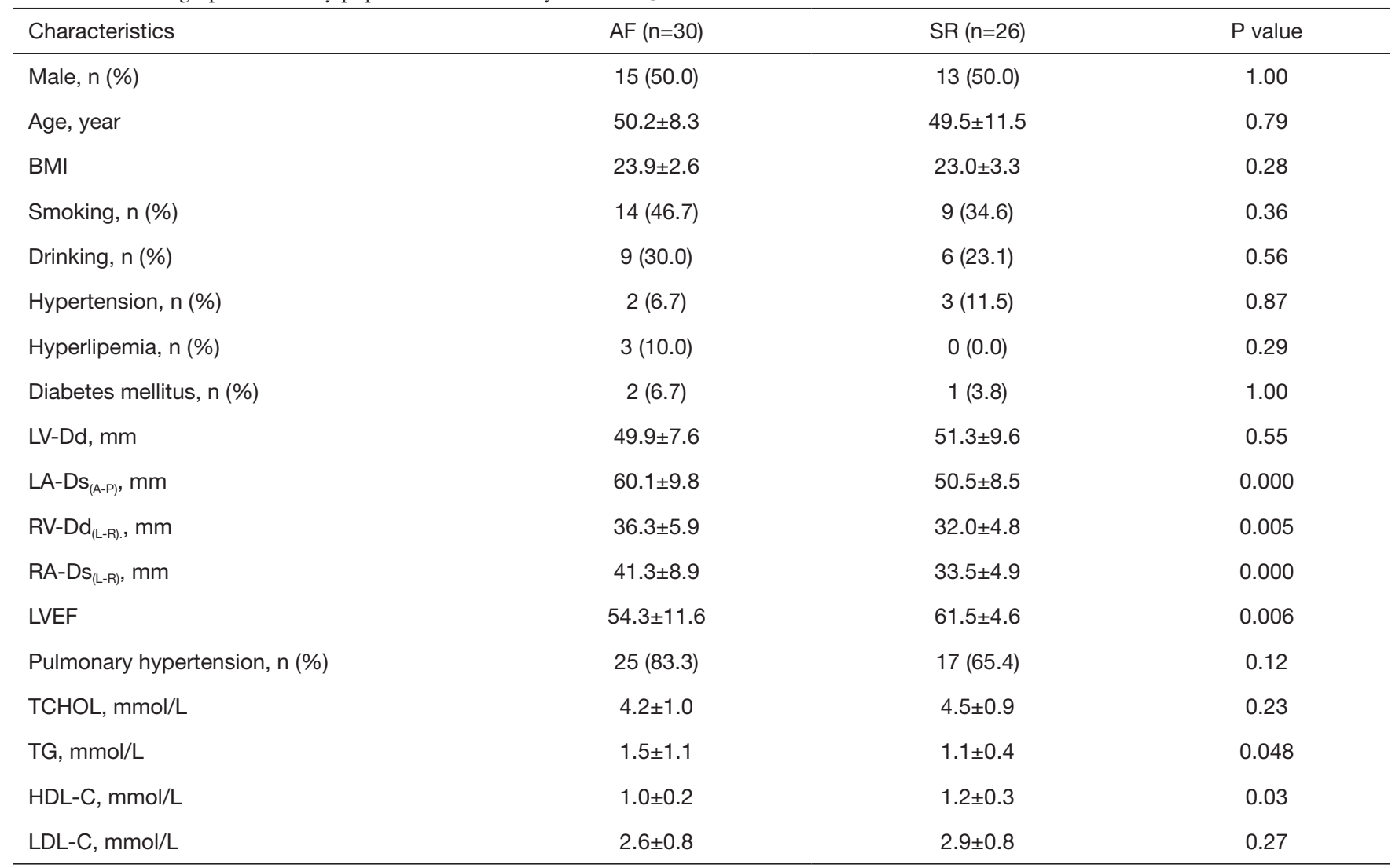

All variables displayed as mean \pm SEM. iTRAQ, isobaric tags for relative and absolute quantification; GC-MS, gas chromatography-mass spectrometer; AF, atrial fibrillation; SR, sinus rhythm; BMI, body mass index; LV-Dd, left ventricular-diastolic diameter; LA-Ds(A-P), left atrium-systolic diameter (anterior-posterior); RV-Dd(L-R), right ventricular-diastolic diameter (left-right); RA-Ds(L-R), right atrium-systolic diameter (left-right); LVEF, left ventricular ejection fraction; TCHOL, total cholesterol; TG, triglyceride; HDL-C, high-density lipoprotein cholesterol; LDL-C, low-density lipoprotein cholesterol; SEM, standard error of the mean. 
Table S2 Demographics of study population in the study of ELISA

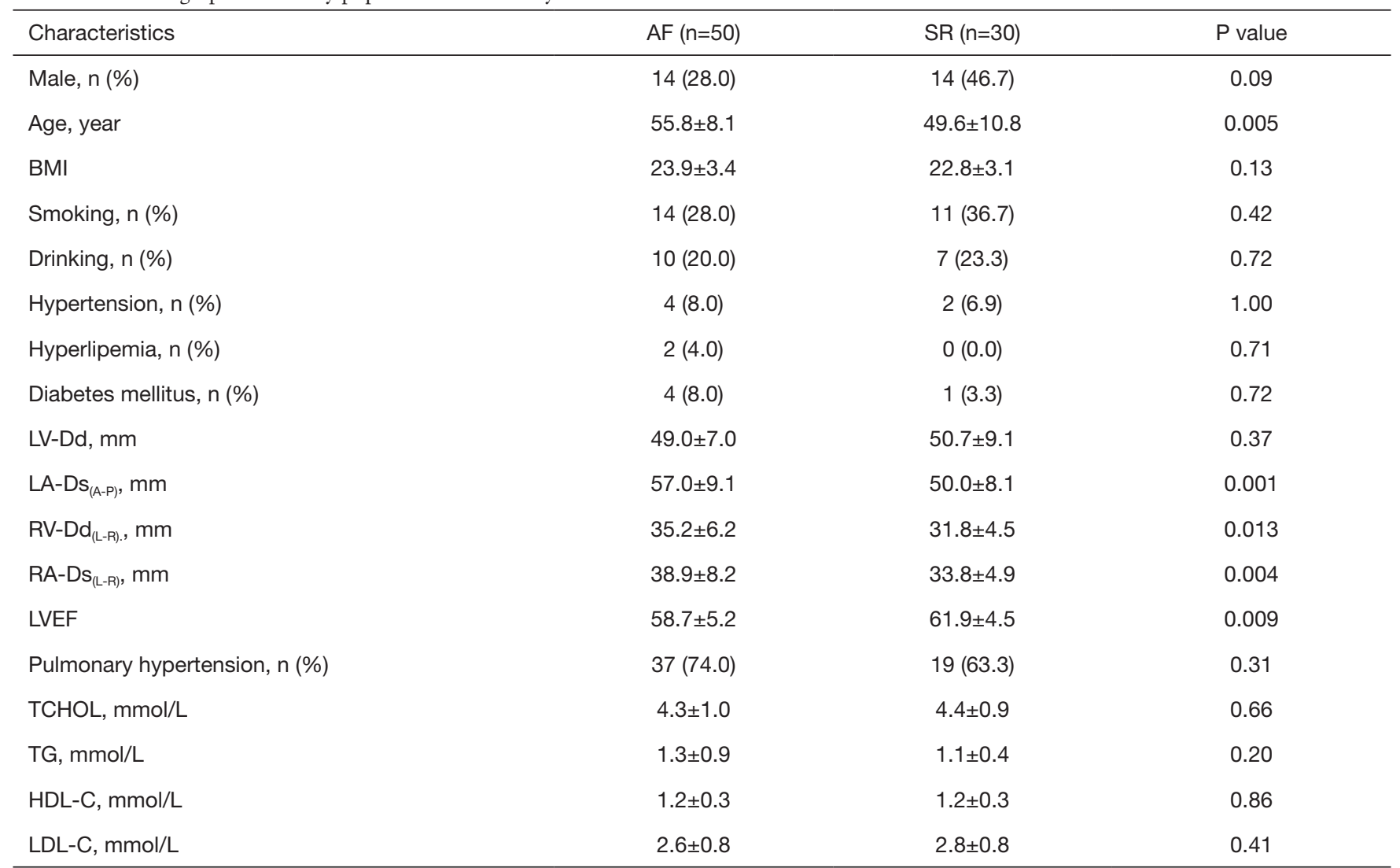

All variables displayed as mean \pm SEM. ELISA, enzyme-linked immunosorbent assay; AF, atrial fibrillation; SR, sinus rhythm; BMI, body mass index; LV-Dd, left ventricular-diastolic diameter; LA-Ds(A-P), left atrium-systolic diameter (anterior-posterior); RV-Dd(L-R), right ventricular-diastolic diameter (left-right); RA-Ds(L-R), right atrium-systolic diameter (left-right); LVEF, left ventricular ejection fraction; TCHOL, total cholesterol; TG, triglyceride; HDL-C, high-density lipoprotein cholesterol; LDL-C, low-density lipoprotein cholesterol; SEM, standard error of the mean. 
Table S3 DPs identified by iTRAQ in plasma samples

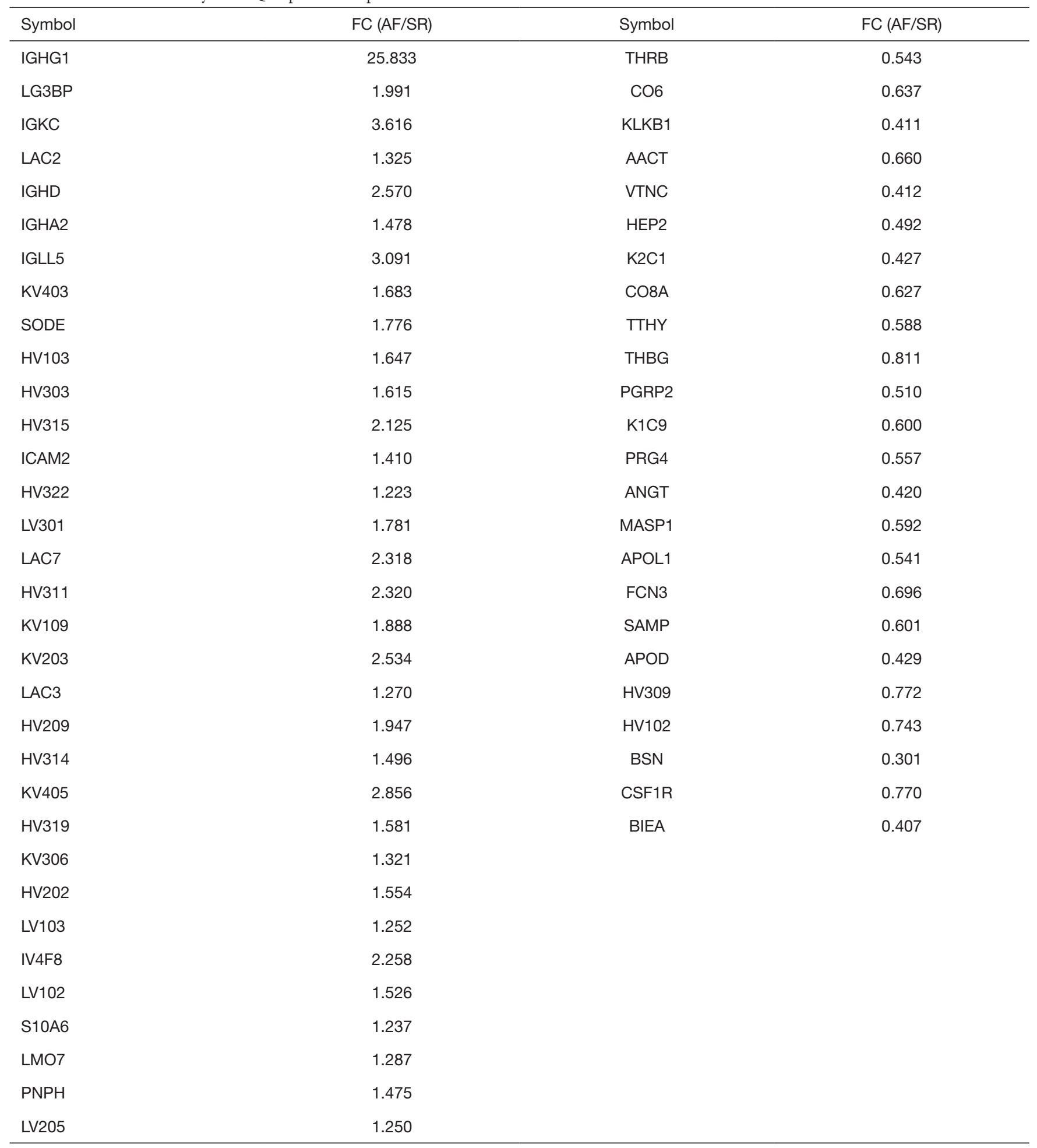

DPs, differential proteins; iTRAQ, isobaric tags for relative and absolute quantification; GC-MS, gas chromatography-mass spectrometer; FC, fold change; AF, atrial fibrillation; SR, sinus rhythm. 
Table S4 DMs distinguished by GC-MS in AF and SR patients in plasma samples

\begin{tabular}{|c|c|c|c|}
\hline Metabolites & $\mathrm{FC}(\mathrm{AF} / \mathrm{SR})$ & VIP & $P$ value \\
\hline Thymidine 5'-monophosphate & 0.055960169 & 4.35825 & 4.50226E-20 \\
\hline D-Altrose & 0.072301309 & 4.1636 & $6.61704 \mathrm{E}-15$ \\
\hline Galactinol & 8.526085448 & 3.54995 & $2.54838 \mathrm{E}-10$ \\
\hline Linoleic acid & 5.133578375 & 1.42721 & $5.05711 \mathrm{E}-09$ \\
\hline Palmitic acid & 2.92289767 & 1.13529 & $3.271 \mathrm{E}-07$ \\
\hline Raffinose & 3.672639357 & 2.78736 & 4.40291E-07 \\
\hline Citraconic acid & 0.152894906 & 2.69137 & $5.25766 \mathrm{E}-07$ \\
\hline Phytosphingosine & 0.587703735 & 2.80858 & $3.61056 \mathrm{E}-06$ \\
\hline $\mathrm{N}$-alpha-Acetyl-L-ornithine & 0.386690837 & 3.02793 & $3.98051 \mathrm{E}-06$ \\
\hline Pentadecanoic acid & 5.032190986 & 2.67135 & $5.42421 \mathrm{E}-06$ \\
\hline (-)-Dihydrocarveol & 9.794830761 & 2.4979 & $2.76024 \mathrm{E}-05$ \\
\hline beta-Glycerophosphoric acid & 0.553812067 & 2.82388 & 7.41314E-05 \\
\hline Glycerol & 0.463294272 & 2.57287 & 0.000117695 \\
\hline 4-Androsten-11beta-ol-3,17-dione & 0.546169665 & 2.22298 & 0.000132546 \\
\hline Guaiacol & 0.368431764 & 2.42314 & 0.000134285 \\
\hline Erythrose & 0.625372928 & 2.50641 & 0.000198983 \\
\hline Carbobenzyloxy-L-leucine degr2 & 0.358601984 & 2.30824 & 0.000365596 \\
\hline O-phosphonothreonine & 1.996050482 & 1.97919 & 0.0003823 \\
\hline Hydantoin, 5-(4-hydroxybutyl)- & 3.783275513 & 2.34158 & 0.000426659 \\
\hline Hypoxanthine & 2.119196166 & 2.09096 & 0.000636647 \\
\hline Sucrose & 3.436732319 & 1.5977 & 0.000790514 \\
\hline N-Acetyl-beta-D-mannosamine & 2.48042303 & 2.09165 & 0.000957718 \\
\hline Sedoheptulose & 4.021531542 & 2.15246 & 0.00113941 \\
\hline 3-Hydroxyflavone & 2.8151879 & 1.78086 & 0.00166879 \\
\hline M-cresol & 0.717116571 & 1.79058 & 0.002835953 \\
\hline Hydrocinnamic acid & 4.384060772 & 1.70452 & 0.003039648 \\
\hline Cyclohexylsulfamic acid & 3.739043866 & 1.85271 & 0.003040377 \\
\hline Galactose & 1.649779532 & 1.644 & 0.003634949 \\
\hline Leucrose & 2.231222736 & 1.76262 & 0.003793272 \\
\hline L-Threose & 0.860696309 & 1.80378 & 0.004950915 \\
\hline 2-Amino-2-norbornanecarboxylic acid & 3.813652323 & 1.56531 & 0.005543599 \\
\hline Succinate semialdehyde & 1.67042504 & 1.46701 & 0.005713745 \\
\hline Cumic acid & 1.697941629 & 1.51777 & 0.006084477 \\
\hline Cellobiose & 11.99964756 & 1.8388 & 0.007029194 \\
\hline Arachidonic acid & 2.428534575 & 1.39718 & 0.009960894 \\
\hline Threo-beta-hydroxyaspartate & 7.939586292 & 1.67525 & 0.010931149 \\
\hline dl-p-Hydroxyphenyllactic acid & 1.774520697 & 1.72497 & 0.011137399 \\
\hline 1-Kestose & 0.705017583 & 1.44337 & 0.011995735 \\
\hline 3-(3-Hydroxyphenyl)propionic acid & 3.788173168 & 1.54174 & 0.012404505 \\
\hline 4-Methylumbelliferone & 2.234238851 & 1.52814 & 0.013906513 \\
\hline Xanthotoxin & 0.638548088 & 1.71556 & 0.014839106 \\
\hline Lactose & 3.171432565 & 1.591 & 0.0150684 \\
\hline Octadecanol & 0.564233061 & 1.28234 & 0.015256356 \\
\hline N-Acetyl-L-phenylalanine & 16.52219043 & 1.65853 & 0.016071066 \\
\hline Synephrine & 1.371721751 & 1.55285 & 0.016552426 \\
\hline D-erythronolactone & 3.265839367 & 1.49591 & 0.018409016 \\
\hline Levoglucosan & 6.85372796 & 1.80614 & 0.019807515 \\
\hline Sitosterol & 1.760365011 & 1.60135 & 0.020508144 \\
\hline $\mathrm{N}-2$-Fluorenylacetamide & 1.770829226 & 1.49582 & 0.021659028 \\
\hline N-Carbamylglutamate & 1.073073155 & 1.11081 & 0.024175094 \\
\hline Heptadecanoic acid & 2.634785578 & 1.13994 & 0.027701354 \\
\hline N-Acetyl-L-aspartic acid & 1.496477986 & 1.28475 & 0.035626135 \\
\hline Lactulose & 9.597210611 & 1.64151 & 0.03598997 \\
\hline Glycocyamine & 1.904311519 & 1.50353 & 0.037441883 \\
\hline 3,4-Dihydroxypyridine & 1.081370306 & 1.43717 & 0.041316434 \\
\hline
\end{tabular}

DMs, differential metabolites; GC-MS, gas chromatography-mass spectrometer; FC, fold change; AF, atrial fibrillation; SR, sinus rhythm;

VIP, variable importance in projection. 
Table S5 Omics studies in AF

\begin{tabular}{lll}
\hline Author/reference & Materials (human or animal) & Omics study \\
\hline Liu et al., (7) & Left and right atrial appendages (human) & Proteomics \\
Tu et al., (29) & Left and right atrial appendage (human) & Proteomics \\
Zhang et al., (30) & Left and right atrial appendages (human) & Proteomics \\
Modrego et al., (31) & Left and right atrial appendage (human) & Proteomics \\
Mayr et al., (8) & Atrial appendage tissues (human) & Metabolomics \& proteomics \\
Alonso et al., (32) & Serum (human) & Metabolomics \\
Jiang et al., (6) & Atrial tissues (human) & Transcriptomics \& proteomics \\
Gao et al., (5) & Plasma (human) & Proteomics \\
Barallobre-Barreiro et al., (33) & Atrial specimens (human) & Glycoproteomics \\
Huang et al., (34) & Atrial appendage tissues (human) & Proteomics \\
De Souza et al., (35) & Left-atrial cardiomyocytes and tissues (dogs) & Proteomics \& metabolomics \\
Ko et al., (36) & Fasting plasma (human) & Metabolomics \\
\hline
\end{tabular}

$\mathrm{AF}$, atrial fibrillation.

\section{References}

29. Tu T, Zhou S, Liu Z, et al. Quantitative proteomics of changes in energy metabolism-related proteins in atrial tissue from valvular disease patients with permanent atrial fibrillation. Circ J 2014;78:993-1001.

30. Zhang $\mathrm{P}$, Wang $\mathrm{W}$, Wang $\mathrm{X}$, et al. Protein analysis of atrial fibrosis via label-free proteomics in chronic atrial fibrillation patients with mitral valve disease. PLoS One 2013;8:e60210.

31. Modrego J, Maroto L, Tamargo J, et al. Comparative expression of proteins in left and right atrial appendages from patients with mitral valve disease at sinus rhythm and atrial fibrillation. J Cardiovasc Electrophysiol 2010;21:859-68.

32. Alonso A, Yu B, Qureshi WT, et al. Metabolomics and incidence of atrial fibrillation in African Americans: the Atherosclerosis Risk in Communities (ARIC) study. PLoS
One 2015;10:e0142610.

33. Barallobre-Barreiro J, Gupta SK, Zoccarato A, et al. Glycoproteomics reveals decorin peptides with antimyostatin activity in human atrial fibrillation. Circulation 2016;134:817-32.

34. Huang WJ, Zhou R, Zeng XR, et al. Comparative proteomic analysis of atrial appendages from rheumatic heart disease patients with sinus rhythm and atrial fibrillation. Mol Med Rep 2011;4:655-61.

35. De Souza AI, Cardin S, Wait R, et al. Proteomic and metabolomic analysis of atrial profibrillatory remodelling in congestive heart failure. J Mol Cell Cardiol 2010;49:851-63.

36. Ko D, Riles EM, Marcos EG, et al. Metabolomic profiling in relation to new-onset atrial fibrillation (from the Framingham Heart Study). Am J Cardiol 2016;118:14936. Erratum in: Am J Cardiol 2017;120:339. 